\title{
NOTE
}

\section{On the Dimension of a Hyperbranched Polymer Synthesized from a Styrene Derivative}

\author{
By Yasuhiro Matsuda, ${ }^{1}$ Motoyasu KobaYAShI, ${ }^{1}$ Atsushi TaKaHARA, ${ }^{1, *}$ Akihiro TanaKA, ${ }^{2}$ \\ Hisato HAYASHI, ${ }^{2}$ Masahiko ANNAKA ${ }^{3}$ and Takahiro SATO ${ }^{4}$
}

KEY WORDS: Hyperbranched Polymer / Polystyrene / Hydrodynamic Radius / SEC-MALS / Light Scattering /

Branched polymers generally have smaller dimensions than those of corresponding linear polymers with the same degree of polymerization, which reduces the solution viscosity and makes the processing of production easier. Moreover, branched polymers possess a lot of chain ends where many functional groups can be introduced, and thus have potential in a variety of commercial applications.

Dendrimers are typical examples possessing the abovementioned branched polymer characters, but they require so many steps of reactions for their preparation that they are unsuitable for industrial usage. Recently, Ishizu et al. have proposed a novel, one-pot reaction scheme to synthesize a hyperbranched polymer (HBP) by photopolymerization of $\mathrm{N}, \mathrm{N}$ diethylamino dithiocarbamoylmethylstyrene (DCS), which can also initiate vinyl polymerization to afford branching points, so-called "inimer" (Scheme 1). They estimated the degree of branching by a kinetic approach ${ }^{2}$ and $\mathrm{NMR}^{3}$ to be as high as ca. $0.3-0.4$.

The molecular characterization of such a HBP is an interesting subject not only industrially but also acedemically. Ishizu and Mori ${ }^{1,4,5}$ measured the intrinsic viscosity $[\eta]$ of their. HBP in toluene, and found that the $[\eta]$ value was smaller than that of the linear PS with the same molecular weight. However, this experimental result does not necessarily indicate that the HBP has a smaller size than that of the linear PS, because the hydrodynamic volume of the polymer chain is not proportional to $[\eta]$ itself but to $[\eta]$ multiplied by the molecular weight $M$, and the HBP and PS should be compared at the same degree of polymerization instead of the molecular weight. When the $[\eta] M$ value calculated from the results of Ishizu and Mori was compared with that for the linear PS with the same degree of polymerization, the former became larger than the latter. This indicates that their. HBP chain is larger than that of the linear PS in solution.

We have recently carried out dynamic light scattering (DLS) measurements for the same HBP, and obtained the hydrodynamic radius being larger than that of the linear PS with the same degree of polymerization (see below). This result is consistent with $[\eta] M$ data of Ishizu and Mori, but seems to be inconsistent with the conventional character of branched polymers.

In this communication, we report static and dynamic light scattering data for this. HBP in tetrahydrofuran (THF) and methyl ethyl ketone (MEK), and argue the chain dimension of the HBP in comparison with linear PS. In the argument, we have noticed that the repeating unit of this. HBP is not identical with that of PS. For PS, the phenyl ring attaches the polymer chain as the side group, but for the HBP the phenylene ring is included into the main chain ( $c f$. Scheme 1).

\section{EXPERIMENTAL}

The HBP was synthesized using DCS as an inimer according to a previous procedure reported by Ishizu and Mori. ${ }^{1}$ The obtained HBP was purified and fractionated by precipitation using a THF/hexane system to remove the oligomers and the polymers with low molecular weight. A hexane was dropwisely added to a THF solution of the HBP to form the suspension of the HBP, which was allowed to stand overnight. Highly viscous solution was obtained by decantation of a portion of supernatant solution. The viscous solution was diluted with THF again to repeat fractionation. Test solutions were prepared by adding THF or MEK to the dried HBP sample and stirring overnight before measurements.

Size-exclusion chromatography on-line multi-angle light scattering (SEC-MALS) measurement was carried out for the HBP using two directly connected poly(styrene-divinylbenzene) gel columns (Shodex KF-805L and KF-807L, $1 \mathrm{~mL} / \mathrm{min}$ ) and $40{ }^{\circ} \mathrm{C}$ THF as the eluent. A THF solution of the HBP of a concentration $c a .2 .5 \times 10^{-3} \mathrm{~g} / \mathrm{cm}^{3}$ was injected into the columns. The intensity of scattering light and the refractive index at each elution volume were recorded with Wyatt DAWN DSP $\left(\lambda_{0}=690 \mathrm{~nm}\right)$ and Tosoh HLC-8220GPC system, respectively. On the other hand, batch measurements of static

\footnotetext{
${ }^{1}$ Institute for Materials Chemistry and Engineering, Kyushu University, 744 Motooka, Nishi-ku, Fukuoka 819-0395, Japan

${ }^{2}$ Synthesis Research Department, Chemical Research Laboratories, Nissan Chemical Industries, Ltd., 722-1 Tsuboi-cho, Funabashi 274-8507, Japan

${ }^{3}$ Deapertment of Chemistry, Kyushu University, 6-10-1 Hakozaki, Higashi-ku, Fukuoka 812-8581, Japan

${ }^{4}$ Department of Macromolecular Science, Osaka University, 1-1 Machikaneyamacho, Toyonaka 560-0043, Japan

*To whom correspondence should be addressed (Tel: +81-92-802-2517, Fax: +81-92-802-2518, E-mail: takahara@cstf.kyushu-u.ac.jp).
} 


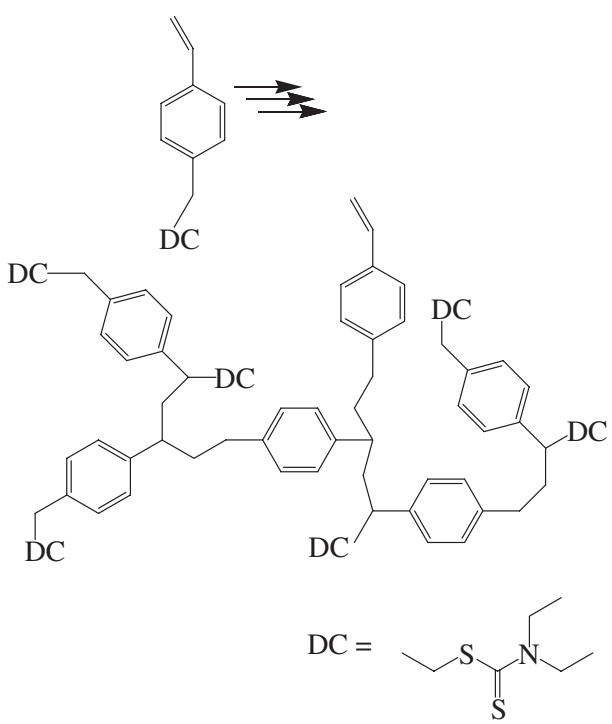

Scheme 1. Chemical structure of the hyperbranched polystyrene synthesized by using $\mathrm{N}, \mathrm{N}$-diethylaminodithiocarbamoylmethylstyrene as an inimer.

light scattering (SLS) and dynamic light scattering (DLS) were carried out with ALV 5000/E/EPP using He-Ne laser as a light source $\left(\lambda_{0}=632.8 \mathrm{~nm}\right)$ at $25^{\circ} \mathrm{C}$. Test solutions for SLS and DLS were filtered with PTFE filter units (DISMIC-13JP) with a pore size of $0.20 \mu \mathrm{m}$ and directly poured into cells in a homemade clean bench.

The specific refractive index increment $(\partial n / \partial c)$ which is necessary for the analysis of SLS and SEC-MALS was measured with Otsuka Electric DRM-1020 at $25^{\circ} \mathrm{C}$. The wavelength of irradiation light $\lambda_{0}$ was $633 \mathrm{~nm}$. The measured $\partial n / \partial c$ of the HBP was $0.233 \mathrm{~cm}^{3} \mathrm{~g}^{-1}$ in THF and $0.254 \mathrm{~cm}^{3} \mathrm{~g}^{-1}$ in MEK, both of which were higher than those of linear PS in the same solvents, probably due to DC moieties. Cauchy dispersion formula shows that there is linear relationship between $\partial n / \partial c$ and $\lambda_{0}^{-2}$, so the dependence of $\partial n / \partial c$ at long wavelength is not so strong. In this study, $\partial n / \partial c$ at $633 \mathrm{~nm}$ was also used for the analysis of SEC-MALS.

\section{RESULTS}

Figure 1 shows SEC chromatograms of refractive index (RI) and light scattering intensity at the scattering angle $\theta=90^{\circ}$ (LS) for the HBP. The maximum concentration of the HBP during the measurement of SEC-MALS was $3.06 \times 10^{-5}$ $\mathrm{g} \mathrm{cm}^{-3}$. Both chromatograms show narrow unimodal peaks suggesting that the HBP molecularly dispersed in THF at such a low concentration. Analyzing the SEC-MALS data, we determined $M_{\mathrm{w}}$ and $M_{\mathrm{w}} / M_{\mathrm{n}}$ to be $9.25 \times 10^{4}$ and 1.5 , respectively. The angular dependence of the scattering intensity was too weak to determine the radius of gyration of the HBP by MALS.

We made batch measurements of dynamic light scattering to obtain the size information of the HBP. When increasing the polymer concentration up to $1 \times 10^{-2} \mathrm{~g} \mathrm{~cm}^{-3}$, we were able to

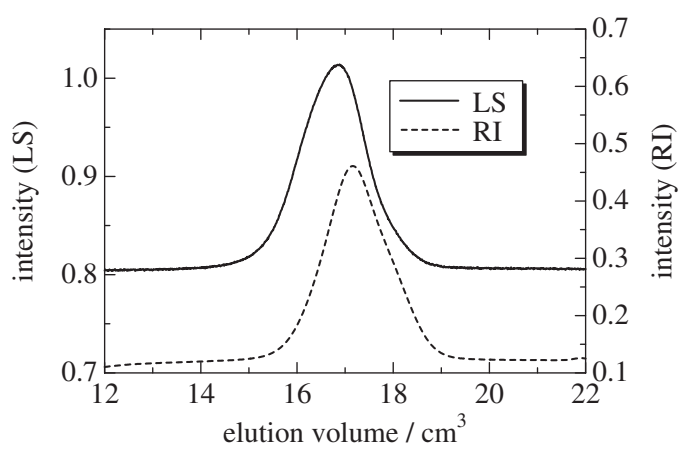

Figure 1. Chromatograms of SEC-MALS measurement for THF solution of the HBP. The maximum concentration during the measurement was $3.06 \times 10^{-5} \mathrm{~g} \mathrm{~cm}^{-3}$.

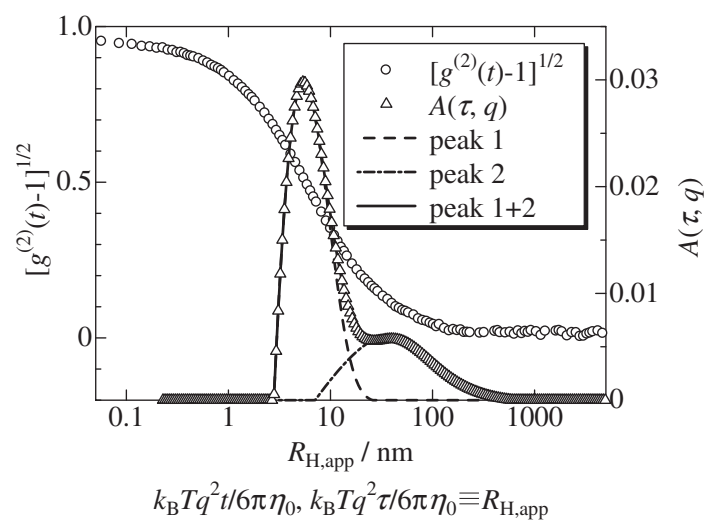

Figure 2. An example of the auto-correlation function and the relaxation time separation spectrum. (THF, $c=1.14 \times 10^{-2} \mathrm{~g} \mathrm{~cm}^{-3}, \theta=105^{\circ}$ ) The auto-correlation function is shown in circles, and the original relaxation spectrum shown in triangles is divided into peak 1 shown in a broken line and peak 2 shown in dot and line.

obtain accurate relaxation-time spectra $A(\tau, q)$, such as shown in Figure 2, where $k_{\mathrm{B}} T q^{2} \tau / 6 \pi \eta_{0}$ (the abscissa) is apparent hydrodynamic radius $R_{\mathrm{H} \text {,app }}$, with $k_{\mathrm{B}} T, q, \tau$, and $\eta_{0}$ being the Boltzmann constant multiplied by the absolute temperature, the magnitude of scattering vector, relaxation time, and the solvent viscosity, respectively. The spectra $A(\tau, q)$ obtained were however bimodal, indicating that the solutions contain small amounts of aggregates of the HBP coexists at such concentrations. Since the linear PS molecularly dispersed in THF at such concentration, ${ }^{6}$ the aggregation of the HBP may be owing to strong interaction DC groups at chain ends of the HBP.

We separated the experimental $A(\tau, q)$ into two peaks, using the empirical trial function $A(\tau, q)=A_{1}(\tau, q)+A_{2}(\tau, q)$ where

$$
A_{\alpha}(\tau, q) \equiv A_{\alpha}\left(\frac{\tau}{\tau_{\mathrm{p}, \alpha}} \mathrm{e}^{-\tau / \tau_{\mathrm{p}, \alpha}}\right)^{h_{\alpha}}
$$

with adjustable parameters $A_{\alpha}, \tau_{\mathrm{p}, \alpha}$, and $h_{\alpha}(\alpha=1,2)$. We used different values of $A_{\alpha}$ and $h_{\alpha}$ at $\tau \leq \tau_{\mathrm{p}, \alpha}$ and $\tau>\tau_{\mathrm{p}, \alpha}$. As illustrated by the solid curve in Figure 2, the experimental $A(\tau)$ were nicely fitted by this trial function. In what follows, we focus on the major fast relaxation component 1 which is expected to be molecularly dispersed HBP. [Note: Experimen- 


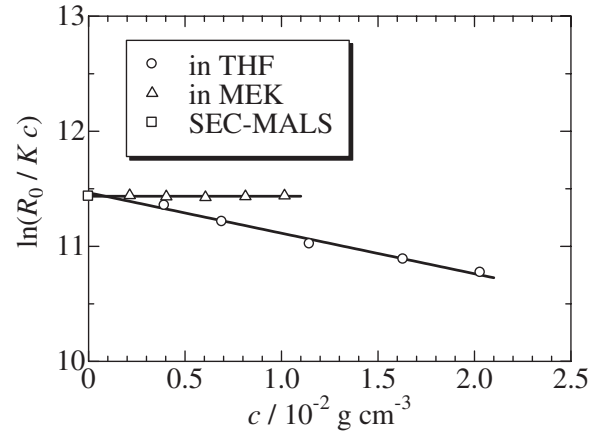

Figure 3. The excess Rayleigh ratio of component 1 (molecularly dispersed HBP) in THF and MEK solutions separated by eqs. 1 and 3 . The inverse of $\ln M_{\mathrm{w}}$ obtained by SEC-MALS is also shown in an open square.

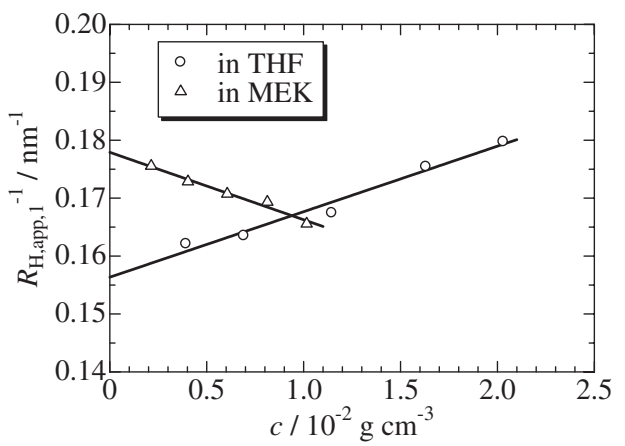

Figure 4. The inverse of the apparent hydrodynamic radius of peak 1 in THF and MEK solutions.

tal results of the slow relaxation component 2 (or the aggregating component of the HBP) are described in the Supporting Information.]

The apparent hydrodynamic radius $R_{\mathrm{H}, \mathrm{app}, 1}$ and the excess Rayleigh ratio $R_{\theta, 1}$ of the major component 1 can be calculated by $^{6}$

$$
\begin{aligned}
& R_{\mathrm{H}, \mathrm{app}, 1} \\
& \quad=\frac{k_{\mathrm{B}} T}{6 \pi \eta_{0}}\left[\lim _{q \rightarrow 0}\left\{\int_{0}^{\infty} A_{1}(\tau, q) \mathrm{d} \tau / \int_{0}^{\infty}\left(q^{2} \tau\right)^{-1} A_{1}(\tau, q) \mathrm{d} \tau\right\}\right]
\end{aligned}
$$

and

$$
R_{\theta, 1}=R_{\theta}\left[\int_{0}^{\infty} A_{1}(\tau) d \tau / \int_{0}^{\infty} A(\tau) d \tau\right]
$$

where $R_{\theta}$ is the total excess Rayleigh ratio obtained by static light scattering. Although not shown, $R_{\theta, 1}$ such obtained was independent of $\theta$, so we estimated $R_{0,1}\left(R_{\theta, 1}\right.$ at $\left.\theta=0\right)$ by averaging $R_{\theta, 1}$ over the entire $\theta$ range examined.

Figures 3 and 4 shows concentration dependences of $\ln \left(R_{0,1} / K c\right)$ and $R_{\mathrm{H} \text {,app,1 }}$ for the HBP in THF (circles). The intercept of $\ln \left(R_{0,1} / K c\right)$ agrees with $\ln M_{\mathrm{w}}$ obtained by SECMALS for a very dilute solution (square). This demonstrates that the peak separation of experimental $A(\tau, q)$ using eq 1 was successfully made and the component 1 corresponds to the molecularly dispersed HBP. [Note: Strictly speaking, the
Table I. Solution properties of the molecularly dispersed HBP measured by SLS, DLS, and SEC-MALS

\begin{tabular}{lcc}
\hline & in THF & in MEK \\
\hline$M_{1}(\mathrm{SEC}-\mathrm{MALS}) / 10^{4}$ & 9.25 & \\
$w_{1} M_{1} / 10^{4}$ & 9.33 & 9.33 \\
$R_{\mathrm{H}, 1} / \mathrm{nm}$ & 6.4 & 5.6 \\
\hline
\end{tabular}

intercept of $\ln \left(R_{0,1} / K c\right)$ is equal to $\ln \left(w_{1} M_{\mathrm{w}, 1}\right)$. However, as explained in the Supporting Information, weight fraction of component $2 w_{2}$ is negligibly small and weight fraction of component $1 w_{1}$ can be approximated to be unity.] In Figure 4, the intercept of $R_{\mathrm{H}, \mathrm{app}, 1}$ gives us the (true) hydrodynamic radius $R_{\mathrm{H}, 1}$ of the molecularly dispersed HBP. (Table I)

Although not shown here, bimodal distributions of $A(\tau, q)$ were obtained also for MEK solutions of the HBP in a similar concentration range. By the same treatment of the static and dynamic light scattering results for the MEK solutions, we obtained $R_{\mathrm{H} \text {,app, } 1}$ and $R_{0,1}$. The results are also shown in Figures 3 and 4 (triangles). The intercept of $\ln \left(R_{0,1} / K c\right)$ agrees with that in THF as expected. While the plot of $\ln \left(R_{0,1} / K c\right)$ against $c$ for THF solutions has a negative slope, the plot for MEK solutions is almost horizontal. This indicates that THF is a good solvent but MEK is a theta solvent for the HBP. Since MEK is a marginal solvent for PS and also cyclohexane, which is a theta solvent for PS, cannot dissolve the HBP even at $50{ }^{\circ} \mathrm{C}$, we can say that the solubility of the HBP is not identical with that of PS maybe due to differences in the repeating unit and chain end. The intercept of $R_{\mathrm{H}, \mathrm{app}, 1}{ }^{-1}$ in MEK is larger than that in THF, indicating $R_{\mathrm{H}, 1}$ in MEK is smaller than that in THF. The HBP may slightly expand in THF by the intramolecular excluded volume.

\section{DISCUSSION}

Our HBP sample has $M_{\mathrm{w}, 1}$ of $9.25 \times 10^{4}$, the weightaverage degree of polymerization $N_{0, \mathrm{w}}$ of 349 , and $R_{\mathrm{H}, 1}$ of $6.4 \mathrm{~nm}$ in THF (a good solvent) and of $5.6 \mathrm{~nm}$ in MEK (a theta solvent) in the molecularly dispersed state at infinite dilution.

Literature $^{7-9}$ gives us the following relations for $R_{\mathrm{H}}$ for linear PS

$$
\frac{R_{\mathrm{H}}(\text { linear PS })}{\mathrm{nm}}= \begin{cases}0.0144 M^{0.561} & \left(\text { in } \mathrm{THF}^{7}\right) \\ 0.0125 M^{0.548} & \left(\text { in } \mathrm{MEK}^{8,9}\right)\end{cases}
$$

Using these relations, we have $R_{\mathrm{H}}($ linear PS) $=5.2 \mathrm{~nm}$ in THF and $4.0 \mathrm{~nm}$ in MEK at $N_{0, \mathrm{w}}=349$. Figure 5 shows the data points of $R_{\mathrm{H}}$ of the HBP and the molecular weight dependence of $R_{\mathrm{H}}$ of linear PS (lin-PS). The hydrodynamic radii of the HBP are larger than those of linear PS with the same $N_{0, \mathrm{w}}$ against the general character of branched polymers. However, it is no wonder, because the backbone chemical structure of the HBP is not the same as linear PS as shown in Scheme 1. The main chains of linear PS consist of carbon-carbon single bond, but those of the HBP consist of carbon-carbon single bond and phenylene ring. That is, we should not compare $R_{\mathrm{H}}$ of the HBP with that of linear PS to investigate the branching effect for polymer dimension. 


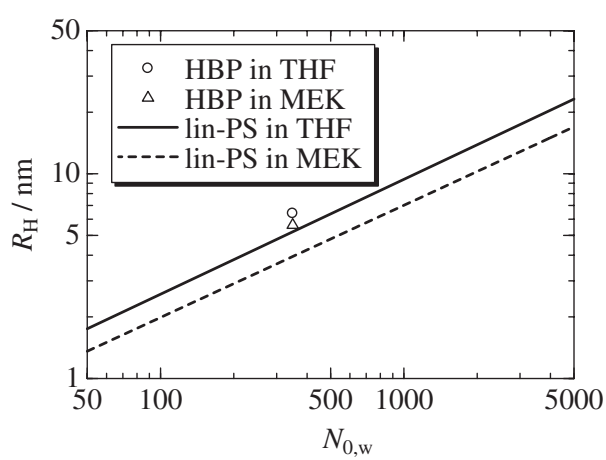

Figure 5. The hydrodynamic radius of the HBP and liner polystyrene (lin-PS) in THF and MEK solutions.

Ishizu and Mori ${ }^{1}$ demonstrated that the intrinsic viscosity $[\eta]$ of HPS is smaller than that of linear PS with the same $M_{\mathrm{w}}$. However, as already mentioned in Introduction, the small $[\eta]$ of the HPS may not mean the shrinkage of the chain due to branching.

The backbone of the HBP includes the phenylene ring, which has much longer virtual bond length $(=0.586 \mathrm{~nm})$ than that of the carbon-carbon single bond $(=0.154 \mathrm{~nm})$. Therefore, the linear polymer chain which should be compared with the HBP must take much more extended conformation than PS. Unfortunately, we have no information about the dimension of such a linear polymer chain at present.

If the HBP is a randomly branched polymer with the degree of branching of 0.3 as reported by Ishizu et al., ${ }^{2,3}$ the g-factor $g_{\mathrm{H}}$ in the unperturbed state is $0.59^{10}$ and $R_{\mathrm{H}}$ of the corresponding linear chain to the HBP must be $9.5 \mathrm{~nm}$ in MEK (a theta solvent). The radius of gyration $\left\langle S^{2}\right\rangle_{0}{ }^{1 / 2}$ of this unperturbed linear chain is expected to be $12.4 \mathrm{~nm}$, if the ratio $\rho$ defined as $\left\langle S^{2}\right\rangle_{0}{ }^{1 / 2} / R_{\mathrm{H}}$ is assumed to be 1.3. (typical value for random coil ${ }^{11}$ ) Using the characteristic ratio $C_{\infty}$, the meansquare bond length $\overline{b^{2}}$, and the number of bonds $N,\left\langle S^{2}\right\rangle_{0}{ }^{1 / 2}$ can be written as

$$
\left\langle S^{2}\right\rangle_{0}=\frac{1}{6} C_{\infty} \overline{b^{2}} N
$$

When we choose $\overline{b^{2}}=\left[2 \times(0.154 \mathrm{~nm})^{2}+(0.586 \mathrm{~nm})^{2}\right] / 3=$ $0.13 \mathrm{~nm}^{2}$, we have $C_{\infty}=6.8$, which is comparable to that for PS $(\sim 10){ }^{7}$

\section{CONCLUSION}

A hyperbranched polymer (HBP) synthesized using $\mathrm{N}, \mathrm{N}$ diethylaminodithiocarbamoylmethylstyrene as an inimer was characterized in tetrahydrofuran (THF) and methyl ethyl ketone (MEK) solutions by light scattering. The following results were obtained:

(1) The hydrodynamic radius of the HBP was larger than that of linear PS with the same degree of polymerization against the general character of branched polymers.

(2) Solvent qualities of MEK and cyclohexane were worse for the HBP than for PS.

(3) In both THF and MEK, the HBP formed a tiny amount of aggregates at concentrations as low as $10^{-2} \mathrm{~g} / \mathrm{cm}^{3}$.

The above features of the HBP may arise from that the repeating unit of the HBP is not identical with that of PS, and also that the HBP bears a number of $N, N$-diethylaminodithiocarbamoyl groups at chain ends.

Electronic Supporting Information Available: Figure S-1 and Table S-1. These materials are available via. the Internet at http://www.spsj.or.jp/c5/pj/pj.htm.

Received: November 19, 2007 Accepted: December 24, 2007 Published: February 15, 2008

\section{REFERENCES}

1. K. Ishizu and A. Mori, Macromol. Rapid Commun., 21, 665 (2000).

2. K. Ishizu, Y. Ohta, and S. Kawauchi, J. Appl. Polym. Sci., 96, 1810 (2005).

3. K. Ishizu, Y. Ohta, and S. Kawauchi, Macromolecules, 35, 3781 (2002).

4. K. Ishizu and A. Mori, Polym. Int., 50, 906 (2001).

5. K. Ishizu and A. Mori, Polym. Int., 51, 50 (2002).

6. M. Kanao, Y. Matsuda, and T. Sato, Macromolecules, 36, 2093 (2003).

7. L. J. Fetters, N. Hadjichristidis, J. S. Lindner, and J. W. Mays, J. Phys. Chem. Ref. Data, 23, 619 (1994).

8. T. A. King, A. Knox, and J. D. G. McAdam, Polymer, 14, 293 (1973).

9. N. C. Ford, Jr., F. E. Karasz, and J. E. M. Owen, Discuss. Faraday Soc., 49, 228 (1970).

10. M. Kurata and M. Fukatsu, J. Chem. Phys., 41, 2934 (1964).

11. H. Yamakawa, "Helical Wormlike Chains in Polymer Solutions," Springer-Verlag, Berlin, 1997. 